\title{
Nanoelectronics: A Primer
}

\author{
Matthew N. O. Sadiku, Yogita P. Akhare and Sarhan M. Musa
}

Roy G. Perry College of Engineering

Prairie View A\&M University

Prairie View, Texas

USA

\begin{abstract}
Nanoelectronics is an offshoot of nanotechnology, the engineering of tiny machines. It is nanotechnology which allows the integration of electronic devices and circuits. It is the art and science of manipulating matter at the nanoscale. The major aim of nanoelectronics is to develop an understanding of the phenomena characteristic of nanometer sized objects. This paper provides a brief introduction to nanoelectronics, its devices, application, benefits, and challenges.
\end{abstract}

Key Words: Nanoelectronics, Nanotechnology, Nanoscale, Nanometer.

\section{INTRODUCTION}

Electronics has evolved rapidly in the last century. The advent of integrated circuits (ICs) has added to compactness and reliability. The electronics industry depends on the ever-decreasing size of transistors, but the scaling cannot continue indefinitely. Nanoelectronics seem to be the most promising successor to lithographic based ICs [1]. The shift from the era of microelectronics to the new era of nanotechnology will make the semiconductors even more pervasive than it is today. Nanotechnology is the study of structures which are in size between 1 to 100 nanometers. Nanoelectronics refer to the applications of nanotechnology in electronics, electronic components, and electronic devices. It covers quantum mechanical properties of materials, semiconductors, nanotubes, nanowires, and so forth.

\section{OVERVIEW OF NANOTECHNOLOGY}

Techniques are now available which make it possible to manipulate materials on the atomic or molecular scale to produce objects which are no more than a few nanometres in diameter. The processes used to make and manipulate such materials are known as nanotechnology, the materials or objects themselves are called nanomaterials, and the study and discovery of these materials is known as nanoscience.

Richard Feymann, the Nobel Prize-winning physicist, introduced the world to nanotechnology in 1959. Nanotechnology involves the manipulation of atoms and molecules at the nanoscale so that materials have new unique properties. It deals with materials, structures, and systems whose components exhibit novel physical, chemical, and biological properties due to their nanoscale sizes. Nanotechnology is a multi-disciplinary field that includes biology, chemistry, physics, material science, and engineering. It is the science of small things at the atomic level or nanoscale level [2].

Nanotechnology seems to be one of the most dominant technologies of the century. It has numerous applications in everyday life, ranging from consumer goods to medicine to improving the environment.

\section{GRAPHENE NANOELECTRONICS}

Silicon-based devices are not be feasible anymore since the scaling limit of silicon is already reached. Carbon seems to be a great alternative to build high performance electronic devices. In electronics, graphene and carbon nanotubes are used as active, semiconducting material. They are potential candidates to replace silicon-based MOSFETs in the future. Carbon nanotubes are hollow cylinders which can be considered as one-dimensional molecules. Graphene is a two-dimensional material 
which represents the special case of a piece of graphite [3]. It consists of a single atomic layer of carbon atoms. Graphene demonstrates good compatibility with the existing semiconductor process. It possesses excellent electronic properties such as high carrier mobility and highly tunable conductance [4]. Its broad industry applications include electronics, optoelectronics. energy, automotive, medical, security, internet of things, mobile convergence, digital manufacturing, and environmental applications.

\section{NANOELECTRONIC DEVICES}

Nanoelectronics is one of the major technologies of nanotechnology. In nanoelectronics, devices are scaled down to nanoscale levels. The major aim of nanoelectronics is to develop an understanding of the phenomena characteristic of nanometer sized objects. Nanoelectronics promises to make computer processors more powerful than are possible with conventional semiconductor fabrication techniques. It provides solutions to how we might increase the capabilities of electronics devices, improving display screens on electronics devices, increasing the density of memory chips, and reducing the size of transistors used in integrated circuits.

\section{APPLICATIONS}

Novel nanoelectronics will provide us with unprecedented levels of functional integration and performance. Some common applications of nanoelectronics include the following [5,6]:

- Consumer electronics: Multimedia and game applications require increasingly higher performances for image rendering.

- Wireless sensor networks: New array devices (e.g., image sensors) require high throughput processing

- Medical electronics: Very large throughput requirement (e.g., image analysis) and very high resilience to transient errors (e.g., Xray)

- Molecular electronics: Perhaps this is the most active field of research. It involves developing individual components that may make up nanoelectronics and could replace the macroscopic silicon components with nanoscale systems. There are so many potential molecules available which can be explored for molecular electronics, e.g. biomolecules: DNA, proteins, and carbon materials: carbon nanotubes, graphene, etc. Examples are molecular diodes, bistable switches, carbon nanotubes, memristors, and nanowire-based electronic devices for logic and memory.

Other applications of nanoelectronics include computers, memory storage, novel optoelectronic devices, displays, quantum computers, radios, energy production, and medical diagnostics.

\section{BENEFITS AND CHALLENGES}

Nanoelectronics is a key enabler for the advancement of information technology. Nanoelectronics technology offers low power, low area, and faster implementation of security protocols. The benefits of dimensional scaling include increased transistor density, lower cost per transistor, and lower switching energy per transistor. As technology scales further into the nanometer regime, it is feasible to implement security primitives using nanoelectronics technologies, such as memristors. Nanoelectronics devices have not only enabled miniature switches for computers but are also used for all kinds of applications including energy conversion and sensing. Nanoelectronics is the next generation approach toward manufacturing of MEMS, microprocessors, optical switching and several other electronic components.

While nanoelectronics promises to provide orders of magnitude improvements in device density, power and performance, some challenges must be addressed to turn the vision into reality. These challenges include the fabrication of largearea $2 \mathrm{D}$ atomic crystals and high-quality $2 \mathrm{D}$ transistors, unreliability due size reduction, and the compatibility between the fabrications and existing semiconductor processes. Because the

investments in CMOS silicon are huge, many resist changes. Since many nanoelectronics devices exhibit their most interesting properties from the microwave frequency up into the optical frequency range, nanoelectronics is a major challenge for the microwave engineering community [7].

\section{CONCLUSION}

Nanoelectronics refer to the use of nanotechnology in electronics. Nanoelectronics technology has become an important component of modern semiconductor manufacturing. It is recognized as a driving force for the information age. With nanotechnology in different forms, new industrial opportunities emerge and create the need for specific competences from students in computer engineering, electrical engineering, and physics. Fabrication of the nanoelectronics devices in the lab is a 
major boost to the student enthusiasm [8,9]. More information about nanoelectronics can be found in the books in [10-15] and the journals

devoted to it:

Journal of Nanoelectronics and Optoelectronics and International Journal of Nanoelectronics and Materials.

\section{REFERENCES}

[1] M. Haselman and S. Hauck, "The future of integrated circuits: A survey of nanoelectronics," Proceedings of the IEEE, vol. 98, no. 1, January 2010, pp. 11-38.

[2] M. N.O. Sadiku, M. Tembely, and S.M. Musa, "Nanotechnology: An introduction," International Journal of Software and Hardware Research in Engineering, vol. 4, no. 5, May. 2016, pp. 40-44.

[3] U. Schwalke et al., "Nanoelectronics: From silicon to graphene," Proceedings of International Conference on Design \& Technology of Integrated Systems in Nanoscale Era, 2012.

[4] R. M. Westervelt, "Graphene nanoelectronics," Science, vol. 320, April 2008, pp. 324-325.

[5] G. D. M. Micheli, "Nanoelectronics: Challenges and opportunities," Proceedings of International Symposium on VLSI Design, Automation and Test, April 2007.

[6] B. Yu and M. Meyyappan, "Nanotechnology: Role in emerging nanoelectronics," Solid-State Electronics, vol. 50, no. 4, April 2006, pp. 536-544.

[7] P. Russer and N. Fichtner, "Nanoelectronics in radio-frequency technology,” IEEE Microwave Magazine, May 2010, pp. $119-135$.

[8] E. Bruun and I. Nielsen, "A model for the development of university curricula in nanoelectronics," European Journal of Engineering Education, vol. 35, no. 6, 2010, pp. 655-666.

[9] V. Kumar and A. Vohra," Nanoelectronics in engineering curricula," IETE Technical Review, vol. 22, no. 1, 2005, pp. 6973.

[10] V. V. Mitin, V. A. Kochelap, and M. A. Stroscio, Introduction to Nanoelectronics: Science, Nanotechnology, Engineering, and Applications. Cambridge University Press, 2008.

[11] S. G. Tan and M. B. A. Jalil, Introduction to the Physics of Nanoelectronics. Woodhead Publishing, 2012.

[12] R. Tsu, Superlattice to Nanoelectrnics. London, UK: Elsevier, $2^{\text {nd }}$ ed., 2011.

[13] R. B. Ismail, M. T. Ahmadi, and S. Anwar (eds.), Advanced Nanoelectronics. Boca Raton, FL: CRC Press, 2013.

[14] R. Waser (ed.), Nanoelectronics and Information Technology: Advanced Electronic Materials and Novel Devices. Weinheim, Germany: Wiley-VCH Verlag, $3^{\text {rd }}$ ed. 2012.

[15] M. Van de Voorde et al. (eds.), Nanoelectronics: Materials, Devices, Applications. Wiley- VCH Verlag, 2017.

\section{AUTHORS}

Matthew N.O. Sadiku is a professor in the Department of Electrical and Computer Engineering at Prairie View A\&M University, Prairie View, Texas. He is the author of several books and papers. His areas of research interests include computational electromagnetics and computer networks. He is a fellow of IEEE.

Yogita P. Akhare is a doctoral student at Prairie View A\&M University, Prairie View, Texas. Her research interests include machine drives and nanotechnology.

Sarhan M. Musa is a professor in the Department of Engineering Technology at Prairie View A\&M University, Texas. He has been the director of Prairie View Networking Academy, Texas, since 2004. He is an LTD Sprint and Boeing Welliver Fellow. 